\title{
Expresión Inmunohistoquímica de Ki-67 en Lesión Central de Células Gigantes
}

\author{
Immunohistochemical Expression of Ki-67 in Central Giant Cell Lesion
}

\author{
Adrian Castro Ortega; Daniela Muela Campos; Uriel Soto Barreras \& Ronell Bologna-Molina
}

CASTRO, A. O.; MUELA, D. C.; SOTO, U. B. \& BOLOGNA-MOLINA, R. Expresión inmunohistoquímica de Ki-67 en lesión central de células gigantes. Int. J. Odontostomat., 13(2):150-156, 2019.

RESUMEN: La lesión central de células gigantes (LCCG) es una lesión osteolítica benigna que en algunos casos presenta un comportamiento agresivo, con recidiva y mal pronóstico. Ki-67 es una proteína nuclear cuya función general es la regulación de la proliferación celular. Este marcador es utilizado para el reconocimiento de células en proliferación y como herramienta de pronóstico en el diagnóstico de neoplasias. El objetivo de este estudio fue cuantificar la inmunoexpresión de Ki-67 en las diferentes poblaciones celulares de las LCCG y analizar su asociación con las características clínicas, demográficas y radiográficas. Se evaluó la inmunoexpresión de Ki-67 de 17 casos de LCCG en dos poblaciones celulares: células gigantes multinucleadas (CGM) y células mesenquimatosas estromales (CME). El análisis estadístico se efectuó con el programa SAS 9.0 y SPSS versión 23.0, con un nivel alfa impuesto de $P<0,05$. Las CME mostraron inmunoexpresión promedio de 9,4 \% y las CGM de 0,65 \%. No se encontró relación estadísticamente significativa entre las características clínicas, demográficas y radiográficas de las LCCG y la inmunoexpresión de Ki-67. La expresión de Ki-67 en CME sugiere que esta población se encuentra en constante actividad celular y que las LCCG son lesiones dinámicas y en constante proceso de diferenciación.

PALABRAS CLAVE: inmunoexpresión, Ki-67, lesión central de células gigantes, proliferación celular.

\section{INTRODUCCIÓN}

La Organización Mundial de la Salud (OMS), define a la lesión central de células gigantes (LCCG) como una lesión osteolítica localizada y benigna, pero ocasionalmente agresiva, formada por tejido fibroso con hemorragia y depósitos de hemosiderina, células gigantes multinucleadas tipo osteoclasto y formación de hueso reactivo (El-Naggar et al., 2017).

Desde su descripción inicial por Jaffe (1953) y hasta la actualidad, su etiopatogénesis, comportamiento clínico, conducta biológica, y muy recientemente su tratamiento no quirúrgico han sido aspectos complejos poco entendidos, que se encuentran aún en exploración.

Las características clínicas, radiográficas e histológicas de la LCCG están bien definidas, pero el papel que juega la expresión de marcadores celulares y moleculares con respecto al comportamiento clínico y respuesta al tratamiento no ha sido completamente explorado (Nogueira et al., 2012).
Ki-67 es una proteína nuclear y nucleolar, cuya función general es la regulación de la proliferación celular (Bullwinkel et al., 2006).

La proteína Ki-67, es utilizada para el reconocimiento de células en proliferación en ensayos inmunohistoquímicos (Gerdes et al., 1983) y como herramienta de pronóstico en el diagnóstico de neoplasias (Stathopoulos et al., 2014). La expresión inmunohistoquímica de la proteína Ki-67 ha sido explorada en la LCCG anteriormente por diversos autores (Kauzman et al., 2004; Fisher et al., 2013), mostrando porcentajes inferiores de los que muestran las lesiones neoplásicas, que van de 1,95 \% a 17,8 \% en células mesenquimatosas estromales (CME) y de 0 $\%$ a menos del $1 \%$ en células gigantes multinucleadas (CGM).

Dichas variaciones pueden deberse a las diferencias en los métodos de conteo celular y la estandarización de los evaluadores. Actualmente la 
posible etiología neoplásica de la LCCG sigue en exploración y se destaca una variabilidad en el grado de expresión inmunohistoquímica de Ki-67, dependiendo las células relacionadas y la agresividad de la lesión (O'Malley et al., 1997).

\section{MATERIAL Y MÉTODO}

Selección de los casos: Se estudiaron 17 casos de LCCG, cuyas muestras histopatológicas son provenientes de los archivos de Laboratorio de Patología Bucal de la Universidad Autónoma MetropolitanaXochimilco, del Servicio de Diagnóstico en Patología Bucal Peribact (México) y del Centro Clínico de Cabeza y Cuello/Hospital Herrera Llerandi, Guatemala.

De las solicitudes de estudio y expedientes clínicos, se obtuvieron datos relativos a edad, sexo, localización, dolor, movilidad dental, deformación, color, imagen radiográfica, resorción radicular, tamaño radiográfico, perforación cortical y desplazamiento dental.

Se incluyeron casos en los que el diagnóstico correspondía a LCCG de acuerdo con la definición propuesta en la Clasificación Histológica de Tumores de la Organización Mundial de la Salud (El-Naggar et al.). Se revisaron las láminas teñidas con hematoxilina y eosina (H\&E) y se realizó la correlación con los aspectos clínicos y radiográficos, para excluir su posible asociación con alguna condición sistémica.

Técnica de Inmunohistoquímica: La técnica de inmunohistoquímica fue estandarizada y llevada a cabo en la Facultad de Odontología de la Universidad Juárez del Estado de Durango, donde se realizó de acuerdo con el siguiente protocolo para el inmunomarcador Ki67 . Se realizaron cortes de $2 \mathrm{~mm}$ de espesor, los cuales fueron montados en laminillas tratadas con poly-Llisina. Los cortes fueron desparafinados en estufa a $45^{\circ} \mathrm{C}$ por 30 minutos y posteriormente colocados en xilol por 5 minutos. Los cortes se hidrataron en un tren de concentraciones decrecientes de alcoholes (100\%, $90 \%, 70 \%$ y $50 \%$ ) y enjuagues con agua destilada.

Para el desenmascaramiento de los epítopes se realizó recuperación antigénica con solución de citrato de sodio $10 \mathrm{mM}(\mathrm{pH} 6,2)$ con olla a presión en microondas a potencia máxima $(750 \mathrm{~W})$ por 5 minutos, dejando enfriar a temperatura ambiente y posteriormente se enjuagó con agua destilada. Las peroxidasas endógenas fueron bloqueadas con peróxido de hidrógeno al 0,9\%, seguidos por lavados con agua destilada y solución salina amortiguada con solución salina de fosfatos pH 7,4 (PBS). Se montaron las laminillas en placas de cubiertas y se colocaron en bastidores para la realización de inmunohistoquímica por capilaridad.

Se incubaron los anticuerpos primarios monoclonales contra Ki-67 (Clona MIB-1, dlución 1:100, Dako Corporation, Carpinteria CA, USA) por un período de $45 \mathrm{~min}$. Posteriormente, los cortes fueron incubados con el segundo anticuerpo biotinilado antiratón/anticonejo y con el complejo streptavidina/ peroxidasa (LSA-B + Labeled streptavidin-biotin, Dako Corporation, Carpinteria CA, USA) por 30 minutos cada uno. Los productos de la reacción fueron visualizados con sustrato de 3,3'-diaminobenzidina-H2O2 (Dako Corporation, Carpintería, CA, USA). Las secciones fueron contrateñidas con hematoxilina de Mayer y las láminas fueron cubiertas con cubreobjetos y resina Entellan.

Evaluación de la inmunoexpresión de Ki-67: Las reacciones de inmunohistoquímica fueron evaluadas en un microscopio de luz convencional por dos investigadores, quienes previamente realizaron un proceso de calibración.

Ki-67 fue evaluado en dos poblaciones celulares: CGM y CME; en esta última se incluyen células ovoidales y fusiformes, correspondientes a células mononucleares, fibroblastos, miofibroblastos y se excluye en la medida de lo posible células endoteliales e inflamatorias.

Para llevar a cabo la evaluación de la expresión inmunohistoquímica de Ki-67 se utilizó el método propuesto por Bologna-Molina et al. (2011), en el cual se seleccionaron tres campos al azar de secciones representativas de cada lesión (Fig. 1).

El conteo de las células positivas se realizó mediante el uso de un objetivo microscópico de 400x, que representa un área de $5,3 \mathrm{~mm}^{2}$. Con la ayuda de una cámara digital, se tomaron microfotografías de las zonas representativas. Luego de tomar las microfotografías de las zonas representativas de cada lesión, estos archivos fueron descargados a una computadora personal y se guardaron como archivos JPG. Cada archivo fue abierto con el programa Microsoft Power Point, se construyó una tabla de 6x6, la cual se colocó sobre la microfotografía y después se guardó de nuevo como archivo JPG (Fig. 2). Pos- 

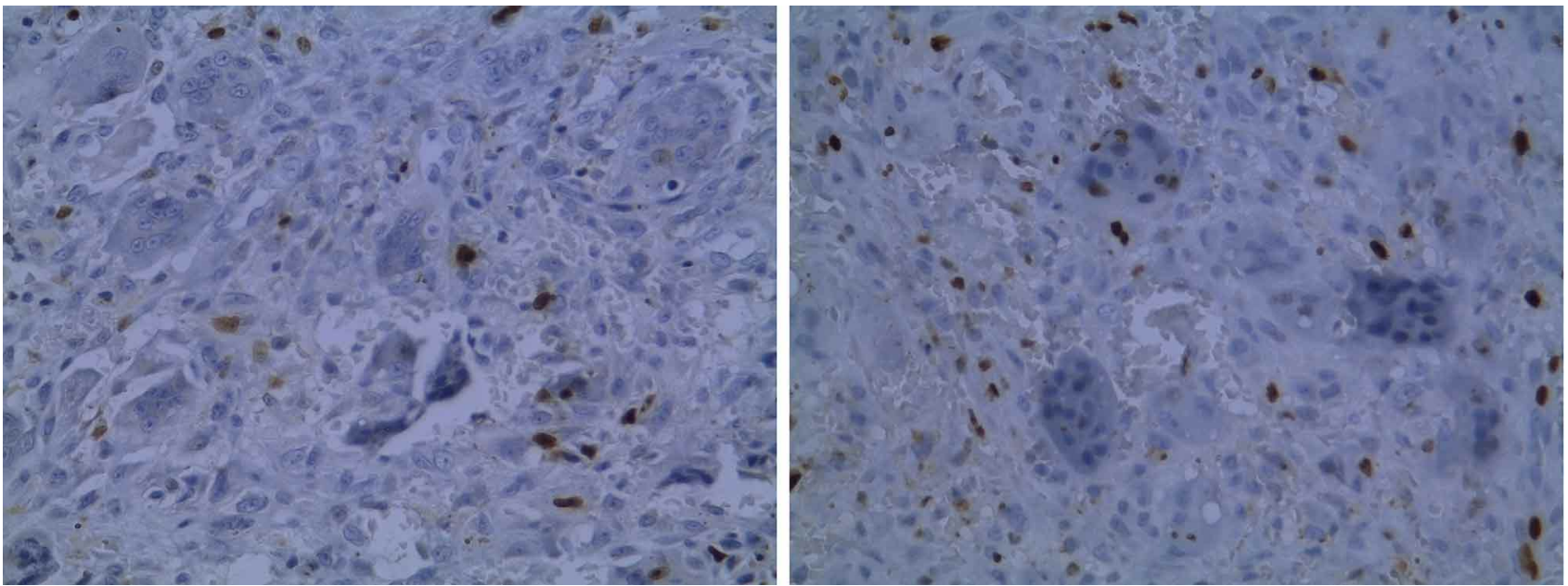

Fig. 1. Inmunoexpresión de KI-67 en CME (400x).

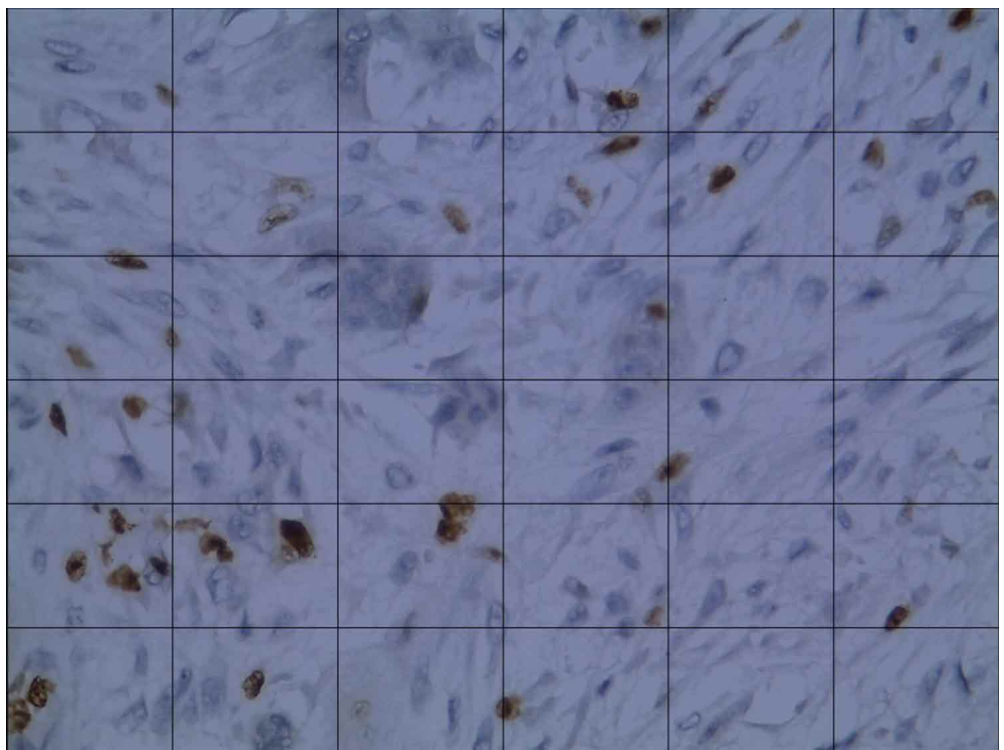

Análisis estadístico: El examinador se estandarizó en todas las variables con un experto a través de Kappa. Todos los datos cuantitativos fueron expresados como media, desviación estándar y rango. Se utilizaron las pruebas Shapiro-Wilk para evaluar la normalidad en la distribución de los datos y Bartlett para la homogeneidad de las varianzas. Se utilizó análisis de varianza (ANOVA) para la expresión de Ki-67. Se analizaron correlaciones entre variables cuantitativas a través de la correlación de Pearson. El análisis se efectuó con el programa SAS 9.0 y SPSS versión 23.0, con un nivel alfa impuesto de $\mathrm{P}<0,05$.

Fig. 2. Tabla Inmunoexpresión de KI-67 en CME (400x)

teriormente, el archivo fue abierto con el programa computacional imagen J (Image J 1.46, aplicación Java para el análisis por imagen), mismo que sirvió como apoyo para llevar a cabo el conteo celular de manera manual. El inicio del conteo celular se realizó desde el marco superior izquierdo y se culminó en el marco superior derecho, siguiendo un mismo orden. Se consideró como expresión inmunohistoquímica positiva toda tinción nuclear visualizada de color café/marrón, de acuerdo con las especificaciones del laboratorio productor del anticuerpo. Se contó el total de células de cada población celular y el número de células teñidas, para de esta manera obtener el porcentaje total de células teñidas por población celular y un porcentaje total de la expresión de cada marcador por lesión.

\section{RESULTADOS}

En el presente estudio se incluyeron un total de diecisiete pacientes con LCCG, seis eran hombres $(35,3 \%)$ y once eran mujeres $(64,7 \%)$ (Relación 1:1,8); el promedio de edad fue de 22.7 años. La localización más afectada fue la mandíbula $(52,9 \%)$ y se identificó movilidad dental en el $76,4 \%$ de los pacientes (13 casos), se presentaron con aspecto multilocular nueve casos $(53 \%)$ y con desplazamiento dental dieciséis casos (94\%) (Tabla I).

De acuerdo con los criterios establecidos por Chuong et al. (1986), mediante una evaluación clínicoradiográfica, se registraron nueve casos (53\%) como 
LCCG agresivas y ocho casos (47 \%) como LCCG con comportamiento no agresivo. Las CME mostraron una expresión inmunohistoquímica promedio de 9,4\%, y escasa expresión en las CGM $(0,65 \%)$. La expresión promedio de Ki-67 en CME fue de 9,3\% en casos agresivos y de $9,5 \%$ en los no agresivos, las CGM mostraron en los casos agresivos una expresión promedio de $0,73 \%$ y de $0,54 \%$ en los casos no agresivos. No se encontraron diferencias estadísticamente significativas en ninguno de los resultados (Tabla II) (Tabla III).

\section{DISCUSIÓN}

Localización

$\begin{array}{llccc}\text { Mandíbula } & 9 & 10,14(2,96) & 6,2-16,8 & 0,5332 \\ \text { Maxilar } & 8 & 8,61(4,57) & 3-16,3 & \end{array}$

Dolor espontáneo

$\begin{array}{lcccc}\mathrm{Si} & 2 & 9,4(0,57) & 9-9,8 & 0,5394 \\ \text { No } & 15 & 9,43(4,02) & 3-16,8 & \end{array}$

Parestesia

$\mathrm{Si}$

No

$$
0
$$

$17 \quad 9,42(3,76) \quad 3-16,8$

Color

\section{Violáceo}

$119,31(3,93)$

$3-16,8$

0,9563

Similar a mucosa

$69,63(3,8)$

$5,4-16,3$

Movilidad dental

$\begin{array}{lcccc}\text { Si } & 13 & 10,12(3,67) & 4,4-16,8 & 0,2572 \\ \text { No } & 4 & 7,18(3,58) & 3-11,6 & \end{array}$

Deformación

$\begin{array}{lcccc}\mathrm{Si} & 17 & 9,42(3,76) & 3-16,8 \\ \mathrm{No} & 0 & ---------\end{array}$

LCCG: Lesión central de células gigantes DE: Desviación estándar, *Prueba estadística ANOVA

Tabla II Distribución y comparación de la expresión de Ki-67 en CME de LCCG de acuerdo con características radiográficas

\begin{tabular}{ccclc}
\hline Variable & $\mathrm{N}$ & Media (DE) & Rango & $\mathrm{P}^{*}$ \\
\hline $\begin{array}{c}\text { Perforación cortical } \\
\mathrm{Si}\end{array}$ & 9 & $9,33(3,64)$ & $4,4-16,3$ & 0,8822 \\
No & 8 & $9,53(4,15)$ & $3-16,8$ & \\
Imagen radiográfica & & & & \\
Unilocular & 8 & $9,99(3,89)$ & $4,4-16,3$ & 0,6798 \\
Multilocular & 9 & $8,92(3,81)$ & $3-16,8$ & \\
Si & & & & 0,9724 \\
No & 5 & $8,7(3,81)$ & $3-12,6$ & \\
Resorción radicular & 12 & $9,72(3,87)$ & $4,4-16,8$ & \\
Si & 16 & $8,96(3,36)$ & $3-16,3$ & 0,3096 \\
No & 1 & $16,8(-)$ & $16,8-16,8$ & \\
Tamaño radiográfico & & & & 0,7463 \\
$\leq 20 \mathrm{~cm}^{2}$ & 14 & $9,33(4,12)$ & $3-16,8$ & \\
$>20 \mathrm{~cm}^{2}$ & 3 & $9,87(1,7)$ & $8,2-11,6$ & \\
\hline
\end{tabular}

LCCG: Lesión central de células gigantes DE: Desviación estándar, *Prueba estadística ANOVA
La LCCG es un tumor benigno de etiológica desconocida. Por tal motivo, se han realizado diversos estudios con la finalidad de explicar su origen y comportamiento biológico, el cual permanece aún incierto.

$\mathrm{Ki}-67$ es un inmunomarcador de proliferación celular y actualmente es comúnmente utilizado como un biomarcador en cáncer de mama, próstata entre otros (Luporsi et al., 2012).

En el presente estudio el porcentaje de expresión en CME fue de 9,4\% y en CGM de 0,65 \%, resultados por debajo de los porcentajes que se presentan en neoplasias malignas (Dowsett et al., 2011). La expresión promedio de Ki67 en las CGM fue de 0,65 \%, una expresión escasa que coincide con lo mencionado por otros autores, quienes no identificaron expresión de Ki-67 en CGM y sugieren que posiblemente las $\mathrm{CME}$ son la población responsable del crecimiento de esta lesión (O'Malley et al.; de Souza et al., 1999; Souza et al., 2000; Kauzman et al.; Kruse-Lösler et al., 2006; Al Sheddi et al., 2014; Kujan et al., 2015). Sin embargo, solo se encontró un estudio, en el que se identificó una fuerte expresión inmuno-histoquímica de $\mathrm{Ki}-67$ en las CGM, exclusivamente en las LCCG consideradas agresivas, indicando que ambas CGM Y CME están activamente involucradas en la actividad proliferativa de estas lesiones (El-Attar et al., 2016). Posiblemente, estos resultados pueden deberse al método de evaluación semicuantitativo. 
Tabla III. Comparación de la expresión de Ki-67 en diferentes poblaciones celulares de LCCG de acuerdo con el comportamiento.

\begin{tabular}{lccccc}
\hline \multirow{2}{*}{ Variable } & \multicolumn{2}{c}{ Agresivo $(\mathrm{n}=9)$} & \multicolumn{2}{c}{ No agresivo $(\mathrm{n}=8)$} & \\
& Media $(\mathrm{DE})$ & Rango & Media $(\mathrm{DE})$ & Rango & $\mathrm{P}^{*}$ \\
\hline CME (Ki67) & $9,34(3,55)$ & $4.4-16,3$ & $9,51(1,5)$ & $3-16,8$ & 0,9303 \\
CGM (KI67) & $0,73(2,2)$ & $0-6,6$ & $0,54(1,52)$ & $0-4,3$ & 0,836 \\
\hline
\end{tabular}

LCCG: Lesión central de células gigantes DE: Desviación estándar, *Prueba estadística ANOVA

La población de CME, la cual incluye células mononucleares, fibroblastos y miofibroblastos, mostraron expresión promedio de Ki-67 de 9,4 \%, resultado similar a lo encontrado en estudios semejantes. Souza et al. refieren una expresión de Ki-67 en LCCG de $1,95 \%$, siendo la de menor expresión de todos los artículos reportados. Por otro lado, Kruse-Lösler et al., en su serie de 26 casos, obtuvieron una expresión de $4.2 \%$ al $4,9 \%$ y O'Malley et al. determinaron una expresión promedio de Ki-67 del 5,6 \%. Se puede argumentar que la diferencia en porcentajes de expresión entre los trabajos reportados y el nuestro se debe a que los autores refieren haber evaluado exclusivamente la respuesta de Ki-67 en células mononucleares, a diferencia de este estudio, en el que se analizó toda la población de CME (mononucleares, fibroblastos, miofibroblastos). Dicha situación se planteó de esta manera, debido a que las características histomorfológicas de estas células pueden plantear una gran dificultad en su diferenciación.

El-Attar et al. reportaron la serie más reciente de expresión de Ki-67 en LCCG con el propósito de determinar si el nivel de expresión puede utilizarse para diferenciar entre LCCG agresivas y no agresivas. En sus resultados mostró que el promedio de células mononucleares positivas a Ki-67 fue 2,3 \% en las LCCG no agresivas y $17,8 \%$ en las agresivas, con una diferencia estadísticamente significativa $(P=0,001)$, y concluyen que la expresión de Ki-67 podría utilizarse para diferenciar el comportamiento biológico de esta lesión. Los resultados obtenidos en este trabajo difieren de lo publicado por El-Attar et al., la expresión promedio de $\mathrm{Ki}-67$ en CME fue de 9,34 \% en los casos agresivos y de $9,51 \%$ en los no agresivos ( $P=0,9303)$.

Actualmente, no se ha identificado un biomarcador confiable que pueda diferenciar entre LCCG agresivas y no agresivas, lo que coincide con el resto de las investigaciones publicadas.

Ki-67 se expresa en todas las fases del ciclo celular, pero no en células en reposo. Considerando que la LCCG es una lesión en constante cambio, el desco- nocer el estado de la lesión (reposo o crecimiento) dificulta determinar el comportamiento biológico - clínico mediante la expresión de Ki-67.

Al igual que en la mayoría de las series reportadas, la edad promedio fue 22,7 años (DE 16,13) y el sexo femenino fue el más afectado (Kruse-Lösler et al.).

De forma similar a lo encontrado en otros estudios (Kruse-Lösler et al.), en este trabajo la localización más común para LCCG fue la mandíbula. Kruse-Lösler et al. reportaron la única serie que analiza otras características clínicas como lo es el porcentaje de dolor y la deformación facial, pero no la comparan con la expresión de Ki-67 en LCCG como si se realiza en nuestro estudio.

Si bien la LCCG no posee una imagen radiográfica específica, la serie más grande reportada hasta la actualidad por Whitaker \& Waldron (1993) (142 casos), encontró que esta lesión tenía una presentación multilocular en el $60 \%$ de los casos, el cual es un porcentaje más alto que lo reportado por otros autores y sólo ligeramente mayor a lo descrito en el presente estudio (53\%).

La presente serie de casos de LCCG a diferencia del resto de los trabajos similares, relacionó la inmunoexpresión de Ki-67 con el mayor número de características clínicas y radiográficas reportadas en la literatura en la actualidad.

Una limitación observada en las diversas series estudiadas es la dificultad para comparar resultados sobre la expresión de los diversos marcadores inmunohistoquímicos en LCCG, debido principalmente a diferencias en los criterios y técnicas para su evaluación.

Otra limitación del presente estudio es el reducido tamaño de la muestra (17 casos), lo cual se puede atribuir, principalmente, a la baja incidencia de LCCG y a la diversidad de tratamientos disponibles para los pacientes, mismos que son seleccionados 
según la experiencia del médico tratante y la disponibilidad de recursos.

El reducido tamaño del grupo de lesiones analizadas sugiere continuar con la evaluación de forma estandarizada de nuevos casos. Sería recomendable, aumentar el tamaño de la muestra, de tal forma que las asociaciones que resultaron marginalmente significativas en esta investigación puedan ser comprobadas o refutadas.

\section{CONCLUSIÓN}

La expresión de Ki-67 en CME sugiere que esta población se encuentra en constante actividad celular y establece una expresión inmunohistoquímica de Ki67 menor a otras patologías de origen neoplásico. Ki67 por si solo no es un marcador confiable para predecir el comportamiento agresivo de la lesión, así mismo, no se encontró relación entre la expresión inmunohistoquímica de Ki-67 y las características demográficas, clínicas y radiográficas de la LCCG.

CASTRO, A. O.; MUELA, D. C.; SOTO, U. B. \& BOLOGNAMOLINA, R. Immunohistochemical expression of Ki-67 in central giant cell lesion. Int. J. Odontostomat., 13(2):150-156, 2019.

ABSTRACT: The central giant cell lesion (CGCL) is a benign osteolytic lesion which in some cases presents an aggressive behavior with recurrence and poor prognosis. Ki67 is a nuclear protein whose general function is the regulation of cell proliferation. This marker is used to identify proliferating cells and as a prognostic tool in the diagnosis of neoplasms. The aim of this study was to quantify the immune expression of Ki-67 in the different cell populations of CGCL and analyze its association with clinical, demographic and radiographic characteristics. The Ki-67 immune expression of 17 cases of LCCG was evaluated in two cell populations: multinucleated giant cells (CGM) and stromal mesenchymal cells (SMC). The statistical analysis was carried out with SAS 9.0 and SPSS version 23.0, with an alpha tax level of $P<0.05$. The CME showed average immune expression of $9.4 \%$ and the CGM of $0.65 \%$. No statistically significant relationship was found between the clinical, demographic and radiographic characteristics of the $\mathrm{CGCL}$ and the immune expression of Ki-67. The expression of Ki-67 in CME suggests that this population is in constant cellular activity, and that the CGCL are dynamic lesions in a continuous differentiation process.

KEY WORDS: immunoexpression, Ki-67, central giant cell lesion, cell proliferation.

\section{REFERENCIAS BIBLIOGRÁFICAS}

Al Sheddi, M. A.; Mosadomi, H. A. \& Al Dayel, F. H. Central giant cell granuloma of the jaws and giant cell tumor of long bones: A clinicopathological, cytometric and immunohistochemical comparative study. Saudi J. Oral Sci.. 1(1):47-53, 2014.

Bologna-Molina, R.; Damián-Matsumura, P. \& Molina-Frechero, N. An easy cell counting method for immunohistochemistry that does not use an image analysis program. Histopathology, 59(4):801-3, 2011.

Bullwinkel, J.; Baron-Lühr, B.; Lüdemann, A.; Wohlenberg, C.; Gerdes, J. \& Scholzen, T. Ki-67 protein is associated with ribosomal RNA transcription in quiescent and proliferating cells. J. Cell. Physiol., 206(3):624-35, 2006.

Chuong, R.; Kaban, L. B.; Kozakewich, H. \& Perez-Atayde, A. Central giant cell lesions of the jaws: a clinicopathologic study. J. Oral Maxillofac. Surg., 44(9):708-13, 1986.

de Souza, P. E.; Paim, J. F.; Carvalhais, J. N. \& Gomez, R. S. Immunohistochemical expression of p53, MDM2, Ki-67 and PCNA in central giant cell granuloma and giant cell tumor. J. Oral Pathol. Med., 28(2):54-8, 1999.

Dowsett, M.; Nielsen, T. O.; A'Hern, R.; Bartlett, J.; Coombes, R. C.; Cuzick, J.; Ellis, M.; Henry, N. L.; Hugh, J. C.; Lively, T.; et al. Assessment of Ki67 in breast cancer: recommendations from the International Ki67 in Breast Cancer working group. J. Natl. Cancer Inst., 103(22):1656-64, 2011.

El-Attar, R. H. M. \& Wahba, O. M. Expression of Ki67, CD31, CD68 and P53 in peripheral and central giant cell granuloma of the jaws. Arch. Cancer Res., 4:2, 2016.

El-Naggar, A. K.; Chan, J. K. C.; Grandis, J. R.; Takata, T.; \& Slootweg, P. J. Pathology and Genetics of Head and Neck Tumors. World Health Organization Classification of Head and Neck Tumours. $3^{\text {rd }}$ ed. Lyon, IARC Press, 2017.

Fisher, G.; Yang, Z. H.; Kudahetti, S.; Møller, H.; Scardino, P.; Cuzick, J.; Berney, D. M. \& Transatlantic Prostate Group. Prognostic value of Ki-67 for prostate cancer death in a conservatively managed cohort. Br. J. Cancer, 108(2):271-7, 2013.

Gerdes, J.; Schwab, U.; Lemke, H. \& Stein, H. Production of a mouse monoclonal antibody reactive with a human nuclear antigen associated with cell proliferation. Int. J. Cancer, 31(1):1320, 1983.

Jaffe, H. L. Giant-cell reparative granuloma, traumatic bone cyst, and fibrous (fibro-oseous) dysplasia of the jawbones. Oral Surg., 6(1):159-75, 1953.

Kauzman, A.; Li, S. Q.; Bradley, G.; Bell, R. S.; Wunder, J. S. \& Kandel, R. Central giant cell granuloma of the jaws: assessment of cell cycle proteins. J. Oral Pathol. Med., 33(3):170-6, 2004.

Kruse-Lösler, B.; Diallo, R.; Gaertner, C.; Mischke, K. L.; Joos, U. \& Kleinheinz, J. Central giant cell granuloma of the jaws: a clinical, radiologic, and histopathologic study of 26 cases. Oral Surg. Oral Med. Oral Pathol. Oral Radiol. Endod., 101(3):34654, 2006.

Kujan, O.; Al-Shawaf, A. Z.; Azzeghaiby, S.; AlManadille, A.; Aziz, K. \& Raheel, S. A. Immunohistochemical comparison of p53, Ki-67, CD68, vimentin, a-smooth muscle actin and alpha-1antichymotry-psin in oral peripheral and central giant cell granuloma. J. Contemp. Dent. Pract., 16(1):20-4, 2015.

Luporsi, E.; André, F.; Spyratos, F.; Martin, P. M.; Jacquemier, J.; Penault-Llorca, F.; Tubiana-Mathieu, N.; Sigal-Zafrani, B.; Arnould, L.; Gompel, A.; et al. Ki-67: level of evidence and methodological considerations for its role in the clinical management of breast cancer: analytical and critical review. Breast Cancer Res. Treat., 132(3):895-915, 2012. 
Nogueira, R. L.; Faria, M. H.; Osterne, R. L.; Cavalcante, R. B.; Ribeiro, R. A. \& Rabenhorst, S. H. Glucocorticoid and calcitonin receptor expression in central giant cell lesions: implications for therapy. Int. J. Oral Maxillofac. Surg., 41(8):994-1000, 2012.

O'Malley, M.; Pogrel, M. A.; Stewart, J. C.; Silva, R. G. \& Regezi, J. A. Central giant cell granulomas of the jaws: phenotype and proliferation-associated markers. J. Oral Pathol. Med., 26(4):15963, 1997.

Souza, P. E.; Mesquita, R. A. \& Gomez, R. sS. Evaluation of p53, PCNA, Ki-67, MDM2 and AgNOR in oral peripheral and central giant cell lesions. Oral Dis., 6(1):35-9, 2000.

Whitaker, S. B. \& Waldron, C. A. Central giant cell lesions of the jaws. A clinical, radiologic, and histopathologic study. Oral Surg. Oral Med. Oral Pathol., 75(2):199-208, 1993.
Dirección para correspondencia:

Adrian Castro Ortega

Laboratorio de Patología Bucal

Facultad de Odontología

Universidad Autónoma de Chihuahua MÉXICO

Email: adrian_caor6@hotmail.com

Recibido : 17-10-2018

Aceptado: 01-02-2019 